PUBLICATION 8

\title{
Antenna effective aperture measurement with backscattering modulation
}

In: IEEE Transactions on Antennas and Propagation 2007.

Vol. 55, No. 10, pp. 2836-2843.

(C) 2007 IEEE.

Reprinted with permission from the publisher.

This material is posted here with permission of the IEEE. Such permission of the IEEE does not in any way imply IEEE endorsement of any of the VTT Technical Research Centre of Finland's products or services. Internal or personal use of this material is permitted. However, permission to reprint/republish this material for advertising or promotional purposes or for creating new collective works for resale or redistribution must be obtained from the IEEE by writing to pubs-permissions@ieee.org. 


\title{
Antenna Effective Aperture Measurement With Backscattering Modulation
}

\author{
Pekka Pursula, Mervi Hirvonen, Kaarle Jaakkola, and Timo Varpula
}

\begin{abstract}
A scattering measurement method for antenna characterization is described. The antenna backscattering is modulated by an oscillator circuit. The modulation begins, when a known RF power is transferred to the oscillator circuit from the antenna. This enables the measurement of the effective aperture of the antenna, from which the antenna bandwidth and radiation pattern are obtained. A theory for antenna aperture measurement is developed using a simple circuit model for the antenna-oscillator system. A dipole and a PIFA with a reactive input impedance at the application frequency were measured. The antenna aperture was measured to an accuracy of $9 \%$, and the measurements complied with simulated and measured references. The method provides simple and accurate bandwidth and radiation pattern measurements with the reactive load the antenna is designed to work with.
\end{abstract}

Index Terms-Antenna measurements, apertures, radar cross sections, scattering.

\section{INTRODUCTION}

$\mathbf{S}_{\mathrm{o}}$ INCE the pioneering work of King in 1949 [1], the study of antenna backscattering has developed into a very diverse field of study. The methods have developed far beyond the measurement of the radar cross section (RCS) of the antenna. Several authors have demonstrated backscattering-based methods for measuring the antenna gain (e.g., [2] and [3]) and the antenna input impedance (e.g., [3] and [4]). In most methods, the antenna under test is connected to known passive loads, but also negative resistance devices have been used [5]. Because the methods require the measurement of the power level of the scattered signal, environmental reflections limit the signal-to-noise ratio and accuracy of the measurement [4].

In this paper, a new approach to backscatter measurement is taken. The antenna under test is connected to an oscillator, which modulates the backscattered signal. This enables measuring the effective antenna aperture (also antenna aperture, or aperture, in this paper) and radar cross section associated with the modulated backscattering.

The oscillator circuit drives a small varactor in the input of the oscillator chip. The oscillator wakes up, when the antenna under test supplies it with enough power. If the RF power required by the oscillator chip is known, the effective antenna aperture can be measured. The method enables a fast and robust way to measure the center frequency, bandwidth and radiation pattern of the antenna under test as was shown, to some extent, in [6].

\footnotetext{
Manuscript received June 1, 2005; revised April 26, 2007. This work was supported by Tekes, The National Technology Agency of Finland.

The authors are with the VTT Technical Research Centre Finland, Espoo FI-02044, Finland (e-mail: pekka.pursula@vtt.fi).

Digital Object Identifier 10.1109/TAP.2007.905821
}

The method has been inspired by the need to characterize small radio frequency identification (RFID) antennas. In RFID applications, the antenna is usually directly matched to a capacitive IC chip. Hence the input impedance of the antenna is inductive, which makes it difficult to measure with conventional transmission techniques. The oscillator IC that is used in the measurements has same input impedance than the RFID chip, which the antenna will actually be used with. This is very important, because high mismatch in the antenna input can affect the current modes in an antenna.

In this paper, measurement results of a dipole and a small PIFA antenna at UHF frequencies are presented. The dipole, with well-known characteristics, is measured, even though its input impedance is nearly real at the application band. To demonstrate the method with a reactive antenna, a PIFA with direct matching to the oscillator chip is measured. The radiation pattern and frequency behavior of the both antennas are measured.

The scattering methods are commonly agreed to give better measurement results than the transmission measurements in the case of small antennas, because there is no feed line to disturb the near fields of the antenna, as is the case with transmission measurements. This is often referred to as a fact, but the effect is rarely demonstrated. The PIFA is designed to be mounted on metal surfaces. The disturbing effect of the feed line can be clearly seen, when the PIFA is measured on different sizes of metal platforms.

This paper proceeds as follows. In the Section II a theoretical model for the antenna-load system is developed and an expression for the effective antenna aperture is derived. The Section II also describes the basic idea of the measurements and considers the effect of different error sources. In Section III measurement results, as well as simulation and reference measurement results are presented.

\section{MeAsurement Method}

\section{A. Theoretical Construction}

To study the scattering effects on a loaded antenna, the model presented in Fig. 1 was used. The antenna and the load are described as impedances $Z_{A}=R_{A}+j X_{A}$ and $Z_{L}=R_{L}+j X_{L}$, respectively. The antenna resistance consists of the radiation resistance $R_{R}$ and the dissipations in the antenna $R_{D}$, i.e., $R_{A}=$ $R_{R}+R_{D}$. The voltage $V$ is the equivalent voltage generated by the incident wave. This model assumes a reciprocal antenna: the current distributions, and thus antenna characteristics, should be identical for transmission and receiving.

Following the reasoning in [7] and [8] expressions for the effective aperture $A_{e}$ and the radar cross section $\sigma$ of the antenna 


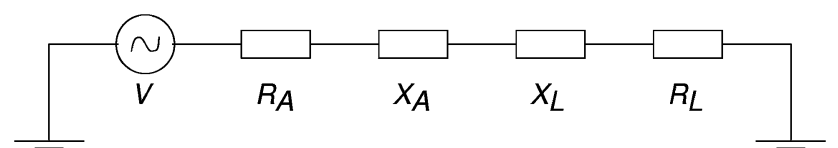

Fig. 1. Series model of a loaded antenna.

can be derived. The RF current $I$ in the circuit can be calculated as

$$
I=\frac{V}{Z}=\frac{V}{\left(R_{A}+R_{L}\right)+j\left(X_{A}+X_{L}\right)}
$$

where $Z$ is the impedance of the circuit. The effective aperture $A_{e}$ is defined as the ratio of the power dissipated in the load resistance $R_{L}$ and the power density $S$ of the incident wave, i.e.

$$
A_{e}=\frac{R_{L} I_{\mathrm{rms}}^{2}}{S}
$$

where $I_{\mathrm{rms}}$ is the effective RF-current in the circuit. Similarly the radar cross section $\sigma$ is defined as the power the antenna radiates, i.e., power dissipated in the radiation resistance $R_{R}$ times the directivity $D$ of the antenna, divided by the power density $S$

$$
\begin{aligned}
\sigma & =\frac{D R_{R} I_{\mathrm{rms}}^{2}}{S}=\frac{\eta D R_{A} I_{\mathrm{rms}}^{2}}{S} \\
& =\frac{G_{A} R_{A} I_{\mathrm{rms}}^{2}}{S} .
\end{aligned}
$$

Here, $\eta$ is the radiation efficiency and $G_{A}$ is the gain of the antenna.

Assuming conjugate matching between the antenna and the load, where $Z_{A}=Z_{L}^{\dagger}$, the maximum power $P_{\max }$ transferred from the antenna to the load can be expressed as

$$
P_{\max }=\frac{V_{\mathrm{rms}}^{2}}{4 R_{A}} .
$$

The power $P_{\max }$ can be identified with the transferred power of the Friis transmission equation

$$
P_{\max }=\frac{G_{A} \lambda^{2}}{4 \pi} S .
$$

Combining (1)-(5), expressions for the effective aperture $A_{e}$ and the radar cross section $\sigma$ become

$$
\begin{aligned}
A_{e} & =\frac{G_{A} \lambda^{2}}{4 \pi} \frac{4 R_{A} R_{L}}{\left(R_{A}+R_{L}\right)^{2}+\left(X_{A}+X_{L}\right)^{2}} \\
\sigma & =\frac{G_{A}^{2} \lambda^{2}}{4 \pi} \frac{4 R_{A}^{2}}{\left(R_{A}+R_{L}\right)^{2}+\left(X_{A}+X_{L}\right)^{2}} .
\end{aligned}
$$

The equations consist of a maximum aperture (first fraction) and a second fraction describing the mismatch between the antenna and the load. Equation (6) can be also expressed with a

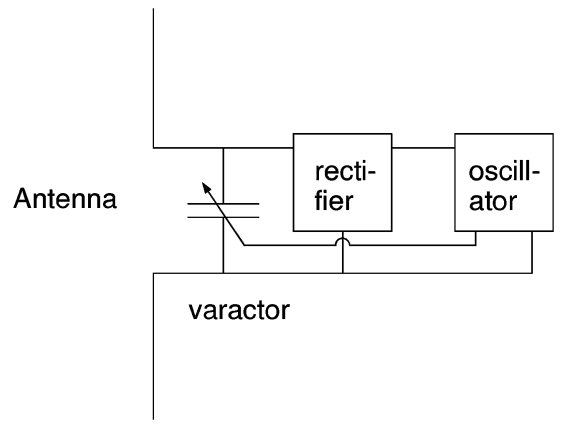

Fig. 2. Block diagram of the antenna—chip system.

reflection coefficient

$$
\begin{aligned}
A_{e} & =\frac{G_{A} \lambda^{2}}{4 \pi}\left(1-|\Gamma|^{2}\right) \\
\sigma & =\frac{G_{A}^{2} \lambda^{2}}{4 \pi}|1-\Gamma|^{2}
\end{aligned}
$$

where the reflection coefficient is defined as

$$
\Gamma=\frac{Z_{L}-Z_{A}^{\dagger}}{Z_{L}+Z_{A}}
$$

and ${ }^{\dagger}$ stands for a complex conjugate.

In previous studies, the measurement methods have been based on measuring the radar cross section. The antenna can also be characterized by measuring the antenna aperture. To do this, the antenna is connected to an oscillator chip, whose input impedance is known. The chip includes a rectifier, a low-frequency oscillator and a varactor, as sketched in Fig. 2. The chip has no battery, but it extracts all the power it needs from the RF power transmitted by an illuminating antenna. The critical RF power $P_{r f, 0}$ the chip requires for the modulation to start, is known.

In this study, two kinds of resonator chips were used, which are described in [8] and [9]. The input impedance of the chips is about $(7-\mathrm{j} 180) \Omega$.

When the oscillator drives the varactor at the input of the chip, the input reactance $X_{L}$ of the chip varies a small amount of $\Delta X$. As seen from (1), a change in chip input reactance modulates the phase of the current in the antenna-chip system. Thus the field scattered from the antenna-chip system is also phase modulated, which is seen as sidebands in the scattered signal. The scattered power is not measured, but only used to indicate the power transferred to the load. Only the transmitted power is measured. This simplifies the measurement apparatus considerably, and enables the center frequency, bandwidth and normalized radiation pattern measurements even in normal laboratory conditions.

Even though it is not necessary to measure the backscattered power, it is interesting to calculate also the radar cross section of the first sideband of the antenna. 


\section{B. Effects of Modulation}

The analysis in Section II-A was based on the assumption that the input impedance of the oscillator chip is constant. Actually the reactance of the chip is modulated by an amount $\Delta X$. What is the effective impedance of the chip, that is actually seen in the measurements?

If the varactor was in the same state all the time, one would measure the antenna aperture given in (6). If the varactor was switched to the other state, one would measure a similar aperture curve, which was shifted to a new center frequency. At a certain frequency, one of the varactor states induces more mismatch to the antenna-chip boundary than the other state. In the higher mismatch state, less RF power is transferred into the chip than in the other varactor state. The oscillator has to get enough DC power to function in the both states of the varactor, for the modulation to function properly.

Without DC capacitors, the chip has to get enough RF power for the oscillator to function in the higher mismatch state of the varactor. In this case, the limiting power $P_{r f, 0}$ would refer to different states of the varactor, and hence to different input impedances of the chip, on different sides of the aperture peak.

But, because the chip has relatively big DC capacitors, the energy charged in the capacitors can be used to drive the oscillator over the higher mismatch state of the varactor. Of course, in the lower mismatch state the chip has to get some extra RF power to charge the capacitors. This averaging behavior leads to a situation, where the critical power $P_{\text {rf }, 0}$ refers to neither of the varactor states, but to a state, which corresponds to the rms-current in the antenna-chip system.

To calculate the rms-current the modulation has to be fixed. For a square wave modulation, the impedance of the antenna-chip system is

$$
Z(t)= \begin{cases}R+j(X+\Delta X) & -T / 2<t<0 \\ R+j(X-\Delta X) & 0<t<T / 2\end{cases}
$$

where $R=\left(R_{A}+R_{L}\right), X=\left(X_{A}+X_{L}\right)$ and $T$ is the period of the modulation. Now the effective current can be expressed as an integral

$$
\begin{aligned}
I_{\mathrm{rms}}^{2}= & \frac{V^{2}}{T} \int_{-T / 2}^{T / 2} \frac{\sin ^{2}\left(\omega_{0} t\right)}{|Z(t)|^{2}} d t \\
= & \frac{V_{\mathrm{rms}}^{2}}{2}\left(\frac{1}{R^{2}+(X+\Delta X)^{2}}\right. \\
& \left.\quad+\frac{1}{R^{2}+(X-\Delta X)^{2}}\right)
\end{aligned}
$$

where $\omega_{0} \gg 1 / T$ is the RF frequency. Now the aperture becomes

$$
A_{e}=\frac{\lambda^{2} G_{A}}{4 \pi}\left(\frac{2 R_{A} R_{L}}{R^{2}+(X+\Delta X)^{2}}+\frac{2 R_{A} R_{L}}{R^{2}+(X-\Delta X)^{2}}\right) .
$$

Especially interesting are the two limits of the mismatch reactance $X$

$$
A_{e}= \begin{cases}\frac{\lambda^{2} G_{A}}{4 \pi} \frac{4 R_{A} R_{L}}{R^{2}+\Delta X^{2}} & X \ll \Delta X \\ \frac{\lambda^{2} G_{A}}{4 \pi} \frac{4 R_{A} R_{L}}{R^{2}+X^{2}} & X \gg \Delta X .\end{cases}
$$

At the sides of the peak, where $|X|$ is big, the modulation does not affect the aperture. But in the middle of the peak, where $X=0$, the aperture is reduced due to the modulation. In other words, the modulation flattens the aperture peak.

Measuring the aperture requires only the measurement of the critical transmit power $P_{t x, 0}$, but it is interesting to study the scattered power also. To derive a theoretical expression for the power in the sidebands, the baseband current in the antenna-chip system is expressed as a Fourier series $I=1 / 2 I_{0}+\Sigma_{n}\left(I_{n, \sin } \sin \left(n \omega_{m} t\right)+I_{n, \cos } \cos \left(n \omega_{m} t\right)\right)$, where $\omega_{m}$ is the modulation frequency. The Fourier coefficients are

$$
\begin{aligned}
& I_{0}=\frac{2 V}{T} \int_{-T / 2}^{T / 2} \frac{d t}{Z(t)} \\
& =4 V \frac{R+j X}{(R+j X)^{2}+\Delta X^{2}} \\
& I_{n, \sin }=\frac{2 V}{T} \int_{-T / 2}^{T / 2} \frac{\sin \left(n \omega_{m} t\right)}{Z(t)} d t \\
& = \begin{cases}\frac{4 V}{n \pi} \frac{j \Delta X}{(R+j X)^{2}+\Delta X^{2}} & n \text { odd } \\
0 & n \text { even }\end{cases} \\
& I_{n, \cos }=\frac{2 V}{T} \int_{-T / 2}^{T / 2} \frac{\cos \left(n \omega_{m} t\right)}{Z(t)} d t \\
& =0 \text {. }
\end{aligned}
$$

The effective RF current corresponding to one of the first sidebands is $I_{\mathrm{rms}}^{2}=1 / 8 I_{1, \sin }^{2}$. Two factors of $1 / 2$ arise because of baseband and RF "root-mean-squaring." The third factor discards power in the other of the first sidebands. Thus the effective current describes power in only one of the symmetric first sidebands. The power in the sideband can also be described by a radar cross section $\sigma_{1}$, which can be calculated from the effective current using (3)-(5)

$$
\sigma_{1}=\frac{\lambda^{2} G_{A}^{2}}{4 \pi} \frac{16}{\pi^{2}} \frac{R_{A}^{2} \Delta X^{2}}{\left(R^{2}+X^{2}+\Delta X^{2}\right)^{2}+4 R^{2} X^{2}} .
$$

\section{Measurement Procedure}

The measurement setup is shown in Fig. 3. It consists of a continuous wave transmitter and a receiver with a spectrum analyzer. The oscillator IC chip is connected to the antenna under test (AUT), which is attached to a rotating mount.

The loaded antenna is illuminated with the transmitter, which creates a power intensity $S$ at the antenna under test. In each 


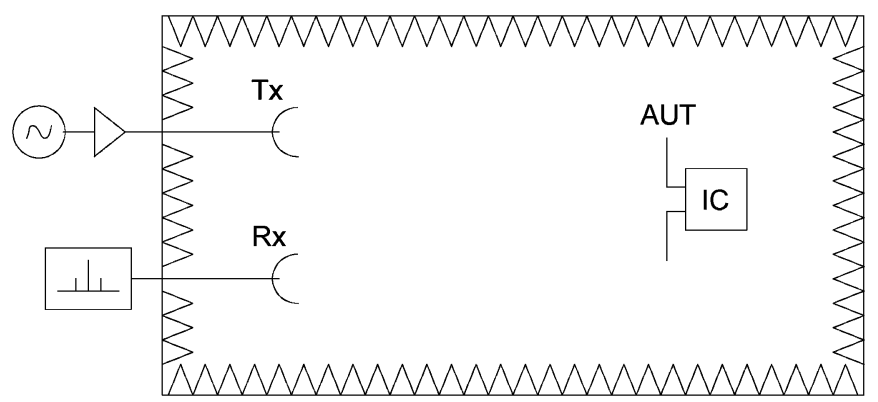

Fig. 3. Measurement setup.

measurement point, the transmitted power $P_{t x}$ is increased until the modulation wakes up, i.e., the sidebands are detected at the receiver. At this critical transmission power $P_{t x, 0}$, the IC chip gets just enough power to function, i.e., the rf power $P_{\text {rf }}$ coupled to the oscillator chip is the known critical power $P_{\mathrm{rf}, 0}$, which the chip needs to function. The critical transmission power $P_{t x, 0}$ is recorded to calculate the antenna aperture. The received power at the sideband frequency $P_{r x, 1}$ was recorded to test (14).

The effective aperture $A_{e}$ and the radar cross section of the first sideband $\sigma_{1}$ are defined as

$$
\begin{aligned}
& A_{e}=\frac{P_{\mathrm{rf}}}{S} \\
& \sigma_{1}=\frac{P_{\mathrm{sc}, 1}}{S}
\end{aligned}
$$

where $P_{\mathrm{sc}, 1}$ is the power scattered from the antenna at sideband frequency.

Because the power intensity $S$ is proportional to the transmitted power $P_{t x}$, it can be measured at a single reference power level $P_{t x, \text { ref }}$, and then scaled

$$
S=\frac{P_{t x}}{P_{t x, \text { ref }}} S_{\text {ref }}
$$

where $S_{\text {ref }}=S\left(P_{t x, \text { ref }}\right)$. The transmitted power is increased until the modulation wakes up. At this power level, the IC chip gets just enough power to function. In other words $P_{\mathrm{tx}}=P_{t x, 0}$ and $P_{\mathrm{rf}}=P_{\mathrm{rf}, 0}$. Now the antenna aperture and the radar cross section can be expressed as

$$
\begin{aligned}
A_{e} & =\frac{P_{\mathrm{rf}, 0}}{S_{\mathrm{ref}}} \frac{P_{t x, \mathrm{ref}}}{P_{t x, 0}} \\
\sigma_{1} & =\frac{(4 \pi d)^{2}}{\lambda^{2} G_{r x}} \frac{P_{r x, 1}}{S_{\text {ref }}} \frac{P_{t x, \mathrm{ref}}}{P_{t x, 0}}
\end{aligned}
$$

where $d$ is the distance between antenna under test and receiver antenna. The power propagation from the antenna under test to the receiver has been calculated with Friis equation. The reference power intensity $S_{\text {ref }}$ can be measured to quite a high accuracy with the same equipment used in the actual measurement.

\section{Error Analysis}

Most antenna scattering measurement methods require the power level measurement of the scattered field. Usually the antenna is connected to a passive load and the scattered field is at the same frequency than the transmitted field. In this kind of a measurements, the transmitted field connects through environment or directly to the receiver, which severely impairs the receiver sensitivity. Thus complicated decoupling and background cancellation systems (e.g., [3]) has been used. In the aperture method described here, the power level of the scattered field is not measured, but only the transmission power, which is much easier to do with high accuracy.

Even if one measures the scattered power, direct coupling is not a problem, because the scattered signal is transferred to sidebands.

On the other hand, the aperture method requires exact knowledge of the required RF power of the oscillator chip. This is not trivial to measure with high accuracy due to the reactive input impedance of the chip. There is also some hysteresis in the rectifier as a function of incoming power. Thus care has to be taken in the measurements, to sweep the transmission power always in the same direction.

Another common error source is the repositioning error in scattering methods, that require antenna under test to be measured with different loads (for example, [3] and [4]). In the aperture method, only one load is used, but the measurement of the reference power intensity at AUT is required. This introduces some repositioning error. But repositioning error gives rise much faster to phase than power level errors [4]. Because in aperture method only power level is measured, repositioning error is not a major problem.

The aperture measurement suffer of course from environmental reflections, which effect the power intensity at antenna under test. But this is taken care of by the reference power intensity measurement.

A quantitative analysis in measurement error can be calculated by using a total differential of the antenna aperture in (17). This yields a relative error of the form

$$
\frac{\Delta A_{e}}{A_{e}}=\frac{\Delta P_{\mathrm{rf}, 0}}{P_{\mathrm{rf}, 0}}+\frac{\Delta P_{t x, \mathrm{ref}}}{P_{t x, \text { ref }}}+\frac{\Delta P_{t x, 0}}{P_{t x, 0}}+\frac{\Delta S_{\mathrm{ref}}}{S_{\mathrm{ref}}}
$$

where prefix $\Delta$ refers to the uncertainty of the quantity.

The power levels can be measured to an accuracy of $0.1 \mathrm{~dB}$ with basic measurement equipment, except the required RF power $P_{\mathrm{rf}, 0}$. This was measured to an accuracy of $6 \%$. Adding up the terms, a relative error of $9 \%$ is acquired.

Similar error analysis can be carried out for the radar cross section of the first sideband in (17). Now there are more error sources, but they can be measured to an accuracy on $0.1 \mathrm{~dB}$, or $1 \%$ quite easily, for required RF power $P_{\mathrm{rf}, 0}$ is not included. Thus, the estimated error is $7 \%$.

\section{MeAsurement Results}

In this section measurements of two antennas are described. First, a dipole antenna is measured due to its well-known characteristics. The oscillator chip used in the measurements has a capacitive input impedance, and thus an external matching circuit is needed. The dipole was measured in an ordinary laboratory environment, to demonstrate the robustness of the measurement system. 


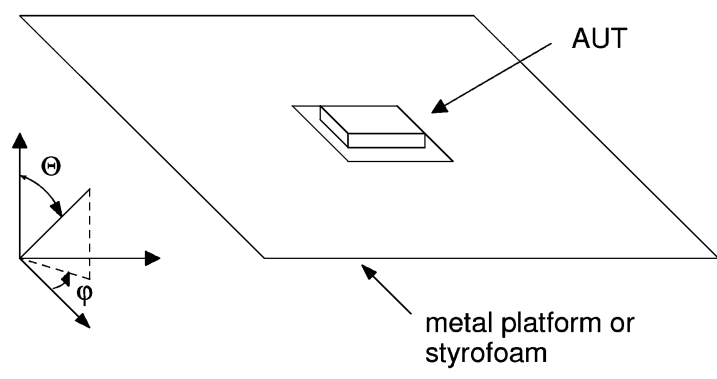

Fig. 4. PIFA (AUT) attached to platform.

The measurement method is well suited for measuring antennas with reactive input impedance. Thus a modified planar inverted antenna (PIFA), with direct impedance matching to the oscillator chip, is measured. The antenna under test is a PIFA that has been designed for RFID applications at $869 \mathrm{MHz}$ band [10]. The antenna is designed to be attached on metal platforms. Hence the measurements were carried out on metal platforms of different sizes, and on a styrofoam support, to simulate the free space condition. The PIFA on an platform is presented in Fig. 4. The PIFA measurements were carried out in an anechoic chamber.

Two kinds of measurements were carried out. In bandwidth measurement, the critical transmitted power $P_{t x, 0}$ was measured at different frequencies. In radiation pattern measurement, the critical power was measured as a function of antenna alignment. First the radiation pattern measurement is considered.

\section{A. Radiation Pattern Measurements}

To measure the normalized power radiation pattern $P_{n}$, one does not have know the reference power intensity $S_{\text {ref }}$ or the critical rf power $P_{\mathrm{rf}, 0}$ in (17). If the measurement setup is stable, these quantities remains constant through the measurement. Because the antenna aperture is the measure of the power transfer between the antenna and the load, the radiation pattern can be calculated as

$$
P_{n}(\varphi, \Theta)=\frac{A_{e}(\varphi, \Theta)}{A_{e, \max }}=\frac{P_{t x, 0, \min }}{P_{t x, 0}(\varphi, \Theta)}
$$

where the $A_{e, \max }=\max \left(A_{e}(\varphi, \Theta)\right)$ is the maximum aperture, which corresponds to the minimum critical transmitted power $P_{t x, 0, \min }=\min \left(P_{t x, 0}(\varphi, \Theta)\right)$. Note that (19) holds exactly only, if the frequency remains constant, as is the case when measuring the radiation pattern.

The error analysis is quite straightforward taking total differential of (19). The uncertainty in the measured power levels was $0.1 \mathrm{~dB}$, which leads approximately to an overall error $\Delta P_{n}=$ $0.2 \mathrm{~dB}$.

The dipole antenna is $15 \mathrm{~cm}$ in length, and resonating at about $870 \mathrm{MHz}$ as such. The antenna is a little shorter than a $\lambda / 2$-dipole, as real implementations of the dipole antenna tend to be. The dipole antenna was connected to the oscillator chip with a $\Pi$-type LC matching circuit. The matching was best at $850 \mathrm{MHz}$, at which frequency the radiation pattern was measured. The measured radiation pattern of the dipole is presented

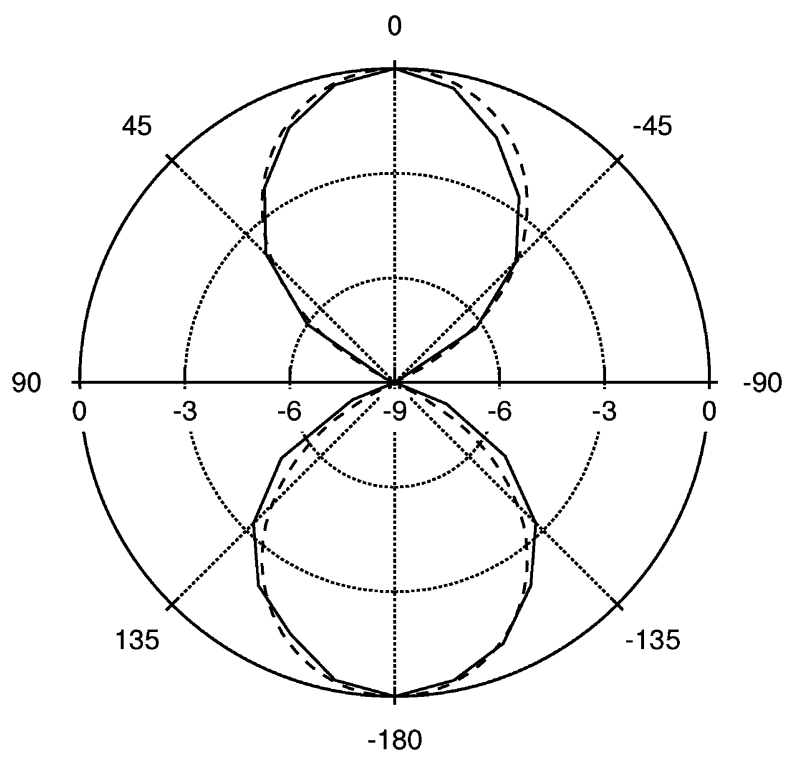

Fig. 5. Measured ( - ) and theoretical (- -) radiation patterns in decibels of the dipole antenna.

in Fig. 5, as well as theoretical radiation pattern of a $\lambda / 2$-dipole from [7]. As can be seen from the figure, the patterns are almost identical.

The measured radiation patterns of both polarizations in a main plane $\left(\phi=135^{\circ}\right)$ of the PIFA are presented in Fig. 6. Both polarizations are scaled to main polarization main beam value. These results can be compared to simulated radiation patterns in Fig. 7 and transmission measurement results in Fig. 8. In the transmission measurements the AUT was fitted to a $50 \Omega$ feed line with a strip line fitting network. To reduce the amount of metallic feed line near the antenna, the fitting network was connected to other measurement apparatus through an optical link. The antenna mounting, including the platform, was identical to the aperture measurement. The antenna was fed through the metal platform.

The results of the transmission measurements differ from the other results significantly, especially in free space, where there is no metal plane to screen the feed line from the antenna. As the radiation patterns become more similar when the size of the metal platform grows, it seems that the feed line and the fitting network disturb the antenna radiation. Thus the transmission measurement results cannot be used as a absolute reference for the aperture method.

The simulated and aperture method radiation patterns are almost identical. Only on the biggest metal platform a difference is seen. The biggest measured metal platform was only $(600 \mathrm{~mm})^{2}$ in area, but simulations were performed on an infinite platform, which explains the difference.

\section{B. Bandwidth Measurements}

The critical power was measured as a function of frequency with a step of a few MHz. The measurements were carried out for the dipole antenna and for the PIFA on different platforms. The antenna aperture was calculated using (17) and the relative error was approximated in Section II-C to be about $10 \%$, mainly from the uncertainty in $P_{\mathrm{rf}, 0}$. 

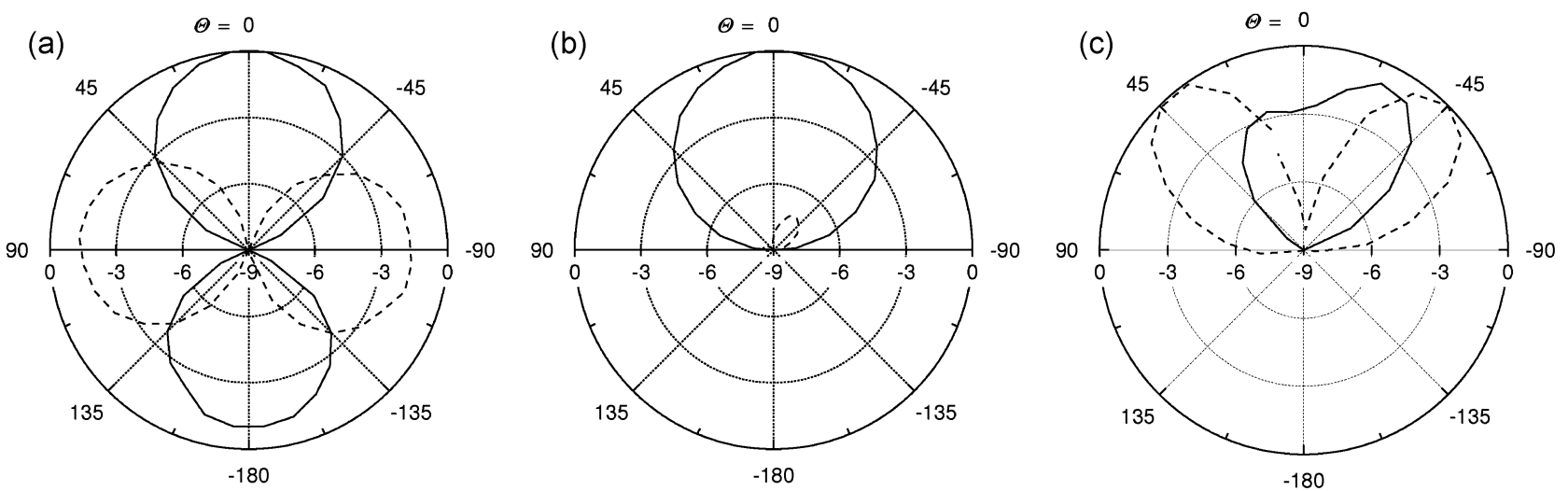

Fig. 6. Radiation patterns in decibels of the PIFA measured with the aperture method: (a) in free space, (b) on ( $150 \mathrm{~mm})^{2}$ metal, and (c) on (600 mm $)^{2}$ metal Solid line $(-)$ present the co-polarization and dashed line (- -) the cross-polarization radiation pattern.
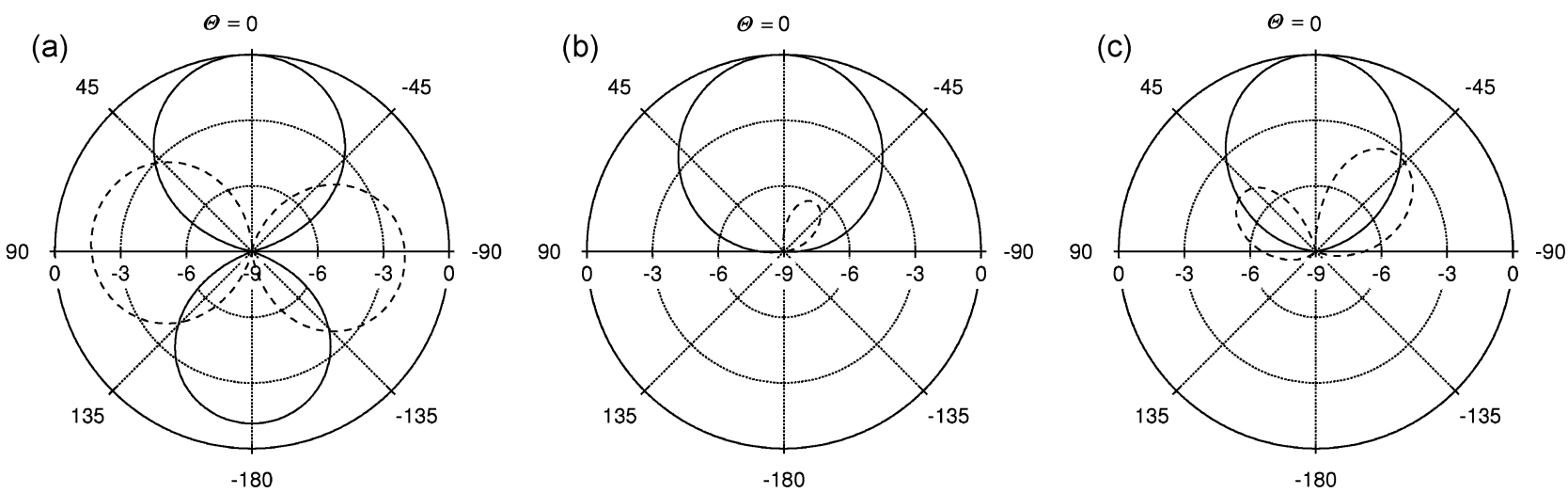

Fig. 7. Radiation patterns in decibels of the PIFA simulated with HFSS: (a) in free space, (b) on $(150 \mathrm{~mm})^{2}$ metal, and (c) on infinite metal. Solid line ( -$)$ present the co-polarization and dashed line (- -) the cross-polarization radiation pattern.
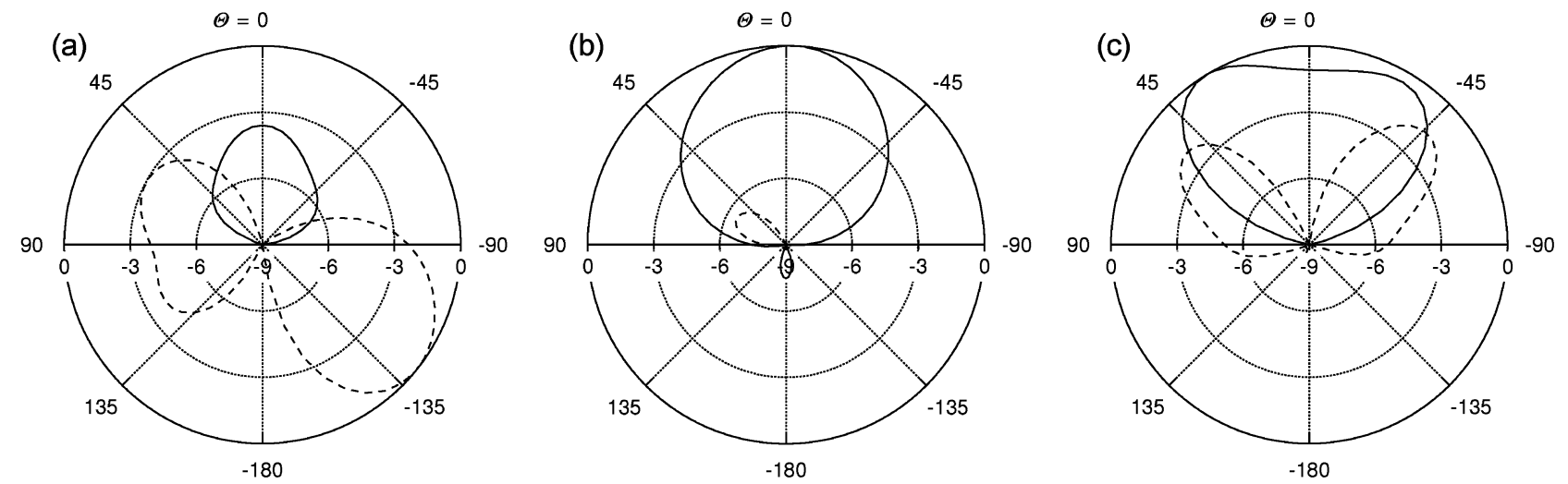

Fig. 8. Radiation patterns in decibels of the PIFA measured with the transmission method: (a) in free space, (b) on $(150 \mathrm{~mm})^{2} \mathrm{metal}$, and (c) on $(600 \mathrm{~mm})^{2}$ metal. Solid line ( -$)$ present the co-polarization and dashed line (- -) the cross-polarization radiation pattern.

To calculate the absolute value of the aperture, the RF power required by the chip has to be known. The required RF power was measured in a test fixture with a network analyzer (VNA). The required power of the oscillator chip used in PIFA measurements was measured to be $P_{\mathrm{rf}, 0}=(16 \pm 1) \mu \mathrm{W}$. This is in line with [8], which describes the chip used.

In the case of the dipole, the oscillator was connected to the VNA with the LC matching circuit used with the antenna. The required power of the chip connected to the matching circuit was measured to be $P_{\mathrm{rf}, 0}=(25 \pm 1) \mu \mathrm{W}$. The input impedance $Z_{\text {in,osc }}$ of the oscillator chip connected to the matching circuit was measured to calculate a reference. The dipole input impedance $Z_{\text {in,dip }}$ is well known, and can be calculated for example from a circuit model presented in [11]. Now the antenna aperture of the dipole can be calculated with (7) and theoretical dipole gain. The measured antenna aperture of the dipole and the calculated reference are presented in Fig. 9.

The matching circuit has a strong effect to the measured peak shape: The matching was designed for an average state of the chip, but the reference does not take into account the modulation. Nevertheless, the curves are almost identical in magnitude and in shape for about $-3 \mathrm{~dB}$ from the aperture maximum. When comparing the curves, one must remember that the relative error in the circuit model is $6 \%$, which is almost enough 


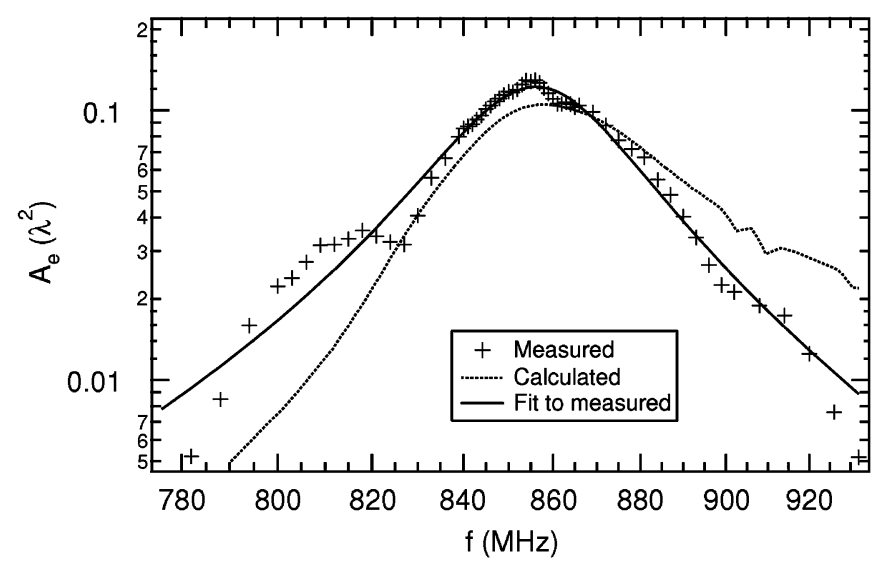

Fig. 9. Measured aperture of the dipole and a calculated reference.

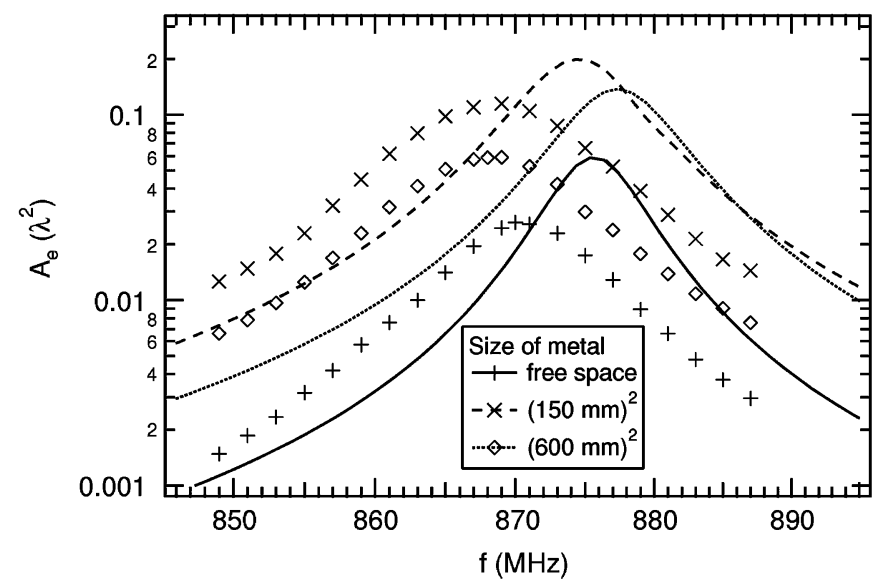

Fig. 10. Measured (markers) and calculated (lines) apertures of the PIFA on different platforms as a function of frequency.

to explain the difference in the curves. The measured maximum aperture at $854 \mathrm{MHz}$ is $0.13 \lambda^{2}$, which is almost exactly the theoretical maximum $[\Gamma=0$ in (6)].

For the PIFA, a reference was calculated in similar way. Values for antenna gain and impedance were simulated with HFSS and measured values of the oscillator chip input impedance were used. The measured and calculated apertures for the PIFA is presented in Fig. 10.

The calculated and measured curves are similar in shape, but shifted in frequency. This can be either a simulator or a measurement error. The simulated peaks are narrower, and about 3 $\mathrm{dB}$ higher than measured ones. This is due to the flattening effect of the modulation, as seen from (12). As the chip reactance is modulated by $\Delta X \approx R_{L}$, a flattening of about $3 \mathrm{~dB}$ is only to be expected.

Also the radar cross section of the first sideband was measured. In Fig. 11 the measured aperture and radar cross section of the PIFA in free space are presented. The uncertainty was estimated in Section II-C, giving $\Delta \sigma_{1} / \sigma_{1}=7 \%$.

The figure illustrates the fact that the effect of mismatch at the antenna-chip interface has a stronger effect to the radar cross section than the aperture. This is also seen in the expressions of $A_{e}$ and $\sigma_{1}$ [(11) and (14)]: the aperture $A_{e} \propto X^{-2}$, but the radar cross section $\sigma_{1} \propto X^{-4}$.

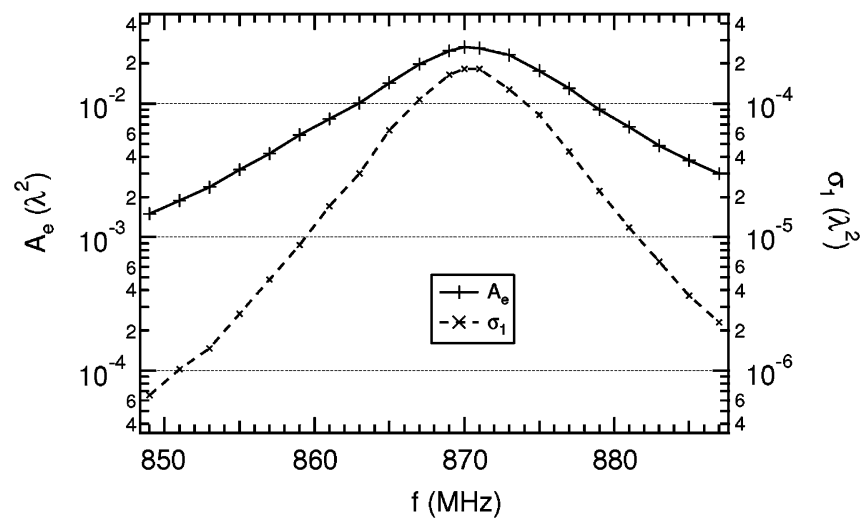

Fig. 11. Measured aperture $A_{e}$ and radar cross section $\sigma_{1}$ of the PIFA in free space.

\section{SUMmARY AND CONCLUSION}

A new method utilizing backscatter data to characterize antenna was presented. The method is based on backscattering modulation, which is achieved by connecting the antenna to a IC chip that consists of an oscillator driven modulator. The method enables the measurement of the antenna aperture and radar cross section of the modulated signal. Because carrier signal coupling from transmitter to receiver does not disturb the measurement, the measurement apparatus is very simple and quite accurate results can be acquired even in normal laboratory conditions.

The method was justified theoretically, and expressions for the antenna aperture and the radar cross section were derived with and without backscattering modulation. It was shown, that the modulator in the antenna load flattens the measured antenna aperture peak.

Radiation pattern and bandwidth measurements are simple and accurate with the introduced method. One reference measurement for the power intensity $S$ is required for determining the absolute value of the antenna aperture, which essentially describes the antenna gain including mismatch at antenna input. From bandwidth measurements, the center frequency and bandwidth can be accurately determined.

A dipole antenna was measured near $869 \mathrm{MHz}$ with the aperture method to demonstrate the method with a well known antenna. The measured radiation pattern was almost identical to the theoretical dipole radiation pattern. The measured frequency behavior was compared to a calculated frequency behavior. In the middle of the peak the curves differed less than $10 \%$ in value, and were of identical shape.

Because the method is well suited for measuring also antennas with reactive input impedance, a reactive RFID antenna with direct impedance matching was measured. The radiation pattern measurement results were similar to simulations and traditional transmission method measurement results, except that the disturbing effect of the feed line when measuring small antennas with transmission measurement technique was clearly seen. The measured center frequency of the PIFA differed from simulated values about $5 \mathrm{MHz}$. The simulated peaks were narrower and higher, which was only to be expected, because of the load modulation.

In general, the introduced method complied very well with the calculated and measured references. 


\section{ACKNOWLEDGMENT}

The authors would like to thank Mr. O. Jaakkola for his contribution to the concept of the study. The IC chips were manufactured by Atmel Germany GmbH.

\section{REFERENCES}

[1] D. D. King, "Measurement and interpretation of antenna scattering," Proc. IRE, vol. 37, pp. 770-777, Jul. 1949.

[2] W. Wiesbeck and E. Heidrich, "Wide-band multiport antenna characterization by polarimetric RCS measurements," IEEE Trans. Antennas Propag., vol. 46, pp. 341-350, Mar. 1998.

[3] J. Appel-Hansen, "Accurate determination of gain and radiation patterns by radar cross-section measurements," IEEE Trans. Antennas Propag., vol. AP-27, pp. 640-646, Sep. 1979.

[4] J. T. Mayhan, A. R. Dion, and A. J. Simmons, "A technique for measuring antenna drive port impedance using backscatter data," IEEE Trans. Antennas Propag., vol. 42, pp. 526-532, Apr. 1994.

[5] R. F. Harrington, "Field measurements using active scatterers," IEEE Trans. Microw. Theory Tech., vol. MTT-11, p. 454, Sep. 1963.

[6] P. Pursula, T. Varpula, K. Jaakkola, and M. Hirvonen, "Antenna radiation characterization by backscattering modulation," in Proc. URSI/ IEEE XXIX Nat. Convention on Radio Science, VTT Symp. 235, Nov. 2004, pp. 115-118 [Online]. Available: http://www.vtt.fi/inf/pdf/symposiums/2004/S235.pdf

[7] J. D. Kraus, Antennas. New York: McCraw-Hill, 1950, pp. 41-56.

[8] U. Karthaus and M. Fischer, "Fully integrated passive UHF RFID transponder IC with 16.7- $\mu \mathrm{W}$ minimum RF input power," IEEE $J$. Solid-State Circuits, vol. 38, pp. 1602-1608, Oct. 2003.

[9] Application note, ATA5590 Tag Antenna Matching [Online]. Available: http://www.atmel.com/dyn/resources/prod_documents/ doc4843.pdf cited Sep. 4, 2006

[10] M. Hirvonen, P. Pursula, K. Jaakkola, and K. Laukkanen, "Planar inverted-F antenna for radio frequency identification," Electron. Lett., vol. 40, pp. 848-850, Jul. 2004.

[11] T. G. Tang, Q. M. Tieng, and M. W. Gunn, "Equivalent circuit of a dipole antenna using frequency-independent lumped elements," IEEE Trans. Antennas Propag., vol. 41, pp. 100-103, Jan. 1993.

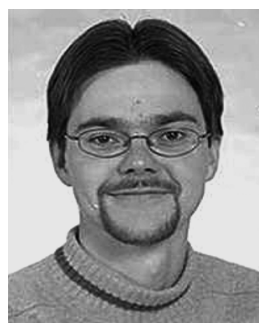

Pekka Pursula was born in Vantaa, Finland, in 1978. He received the Master of Science (Tech.) degree (with distinction) in technical physics from Helsinki University of Technology (TKK), Espoo, Finland, in 2002.

In 2002, he was with Philips Medical Systems Finland developing RF systems in magnetic resonance imaging. Since 2003, he has been with the VTT Technical Research Centre of Finland, Espoo. His present research interests include RFID systems and wireless sensors.
Mr. Pursula received the Young Scientist Award at the URSI/IEEE XXIX Convention on Radio Science, Espoo, Finland, in November 2004.

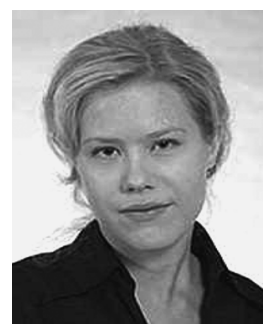

Mervi Hirvonen received the Master of Science (Tech.) and Licentiate of Science (Tech.) degrees in electrical engineering from Helsinki University of Technology (TKK), Espoo, Finland, in 2004 and 2006, respectively.

Since 2002, she has been with the VTT Technical Research Centre of Finland, Espoo, initially as a Research Trainee and, since 2004, as a Research Scientist. Her current research interests include antennas and electromagnetics related to wireless sensors, RFID systems and mobile communications.

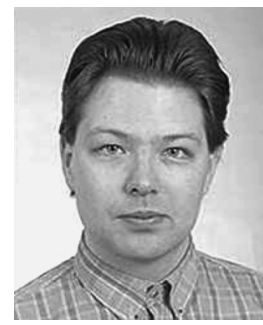

Kaarle Jaakkola was born in Helsinki, Finland, in 1976. He received the Master of Science (Tech.) degree in electrical engineering from Helsinki University of Technology (TKK), Espoo, Finland, in 2003.

Since 2000, he has been working with the VTT Technical Research Centre of Finland, Espoo, first as a Research Trainee and, since 2003, as a Research Scientist. From 2000 to 2002, he participated in the Palomar (EC IST) project developing RF parts for a new RFID system. His current research interests include RFID systems, wireless and applied sensors, antennas, electromagnetic modelling, and RF electronics.

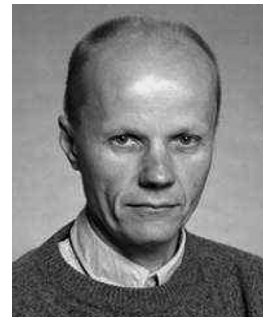

Timo Varpula was born in Seinjoki, Finland, in 1954. He received the Doctor degree from Helsinki University of Technology (TKK), Espoo, Finland, in 1982.

At TKK, he conducted research on weak magnetic fields produced by the human brain, eye, and heart. $\mathrm{He}$ also developed superconducting magnetometers and data analysis for biomagnetic signals. From 1983 to 1986, he worked as a Project Manager at Instrumentarium Corporation, Ltd., where he developed whole-body nuclear magnetic resonance imaging systems. After joining the VTT Technical Research Centre of Finland, Espoo, he was Section Leader from 1986 to 1994 and conducted research on measurement devices for metrological and industrial purposes. In 1994 to 2004, he worked as a Group Manager at VTT Automation and VTT Information Technology. In 2004 to 2006, he was the Research Manager at VTT Microsensing. He is presently Technology Manager at VTT Sensors. His research interests include radiofrequency identification, RF, microwave, and wireless sensors for scientific and industrial applications. He has coauthored 32 scientific publications and holds seven patents. 